Article

\title{
Entropy Analysis on Electro-Kinetically Modulated Peristaltic Propulsion of Magnetized Nanofluid Flow through a Microchannel
}

\author{
Muhammad Mubashir Bhatti ${ }^{1}$, Mohsen Sheikholeslami ${ }^{2, *}$ and Ahmed Zeeshan ${ }^{3}$ \\ 1 Shanghai Institute of Applied Mathematics and Mechanics, Shanghai University, Shanghai 200072, China; \\ mubashirme@yahoo.com \\ 2 Department of Mechanical Engineering, Babol Noshirvani University of Technology, Babol 47148-71167, Iran \\ 3 Department of Mathematics, International Islamic university Islamabad, Islamabad 44000, Pakistan; \\ ahmad.zeeshan@iiu.edu.pk \\ * Correspondence: m.sheikholeslami@stu.nit.ac.ir or mohsen.sheikholeslami@yahoo.com; \\ Tel.: +98-911-396-8030
}

Received: 4 August 2017; Accepted: 7 September 2017; Published: 9 September 2017

\begin{abstract}
A theoretical and a mathematical model is presented to determine the entropy generation on electro-kinetically modulated peristaltic propulsion on the magnetized nanofluid flow through a microchannel with joule heating. The mathematical modeling is based on the energy, momentum, continuity, and entropy equation in the Cartesian coordinate system. The effects of viscous dissipation, heat absorption, magnetic field, and electrokinetic body force are also taken into account. The electric field terms are helpful to model the electrical potential terms by means of Poisson-Boltzmann equations, ionic Nernst-Planck equation, and Debye length approximation. A perturbation method has been applied to solve the coupled nonlinear partial differential equations and a series solution is obtained up to second order. The physical behavior of all the governing parameters is discussed for pressure rise, velocity profile, entropy profile, and temperature profile.
\end{abstract}

Keywords: magnetic field; electrokinetic; peristaltic pumping; nanofluid; perturbation solution; microchannel

\section{Introduction}

During the recent few decades, Nanotechnology has received a remarkable attention due to its numerous applications in industry, since material containing nano-sized particles exhibits various chemical and unique physical features. Major applications include hybrid power engines, fuel cells, microelectronics, domestic refrigerators, pharmaceutical processes, chillers, cooling of nuclear reactors, space technology, boiler flue gas temperature control, and grinding. Nanofluid is the interaction of Nano-sized particles within the fluid. Nanofluid heat transfer is very much favorable to enhance the heat transfer and thermal conductivity of the fluid up to $40 \%$. Nanofluid consists of nano-sized particles (diameter: 1-100 nm) in different Newtonian and non-Newtonian fluid such as water, ethylene glycol, and oil. In nanofluid, nanoparticles are made up of carbon or carbide nanotubes, oxides, and metals. Most of the fluids have low thermal conductivity and thus they are unable to match today's need of cooling rate. For this purpose, a new way to enhance the thermal conductivity of such type of fluids is by adding nano-sized particles in the base fluids (water) to form slurries. Nanofluid has become now the major attention of various researchers for the new manufacturing of automotive and plant cooling systems, and for transfer of heat in different heat exchanger devices. Choi [1] was the first who described experimentally the thermal conductivity of fluid with nanoparticles. Khanafer et al. [2] described the heat transfer enhancement in a two-dimensional enclosure using nanofluid under the influence of buoyancy force. Oztop et al. [3] investigated numerically the 
natural convection in a partially heated rectangular enclosure with nanofluid models. Sheremet and Pop [4] discussed the natural convection in the presence of sinusoidal temperature distributions on nanofluid flow propagating through a porous medium. He modeled the governing flow with the help of Darcy-Boussinesq approximation and the Buongiorno's nanofluid model. They used a second order finite difference method to obtain the numerical solutions. In this study, they found that the Sherwood number and Nusselt number behave as an increasing function due to greater influence of the buoyancy-ratio parameter, thermophoresis parameter, and Rayleigh number, and behave conversely for the Brownian motion parameter and Lewis number.

Xiao et al. [5] presented an analytical model for effective thermal conductivity of nanofluid with Brownian motion effects of nanoparticles. They found that the greatest influence of the concentration of nanoparticles is to enhance the thermal conductivity of nanofluids. Moreover, they also observed that the effective thermal conductivity of nanofluids for the smaller magnitude of nanoparticles is higher than the larger size at a given concentration. Sheremet et al. [6] discussed numerically the transient natural convection through a porous wave wall cavity filled with nanofluid. He used the mathematical model proposed by Tiwari and Das and utilized the Darcy-Boussinesq approximation with thermal dispersion. Cai et al. [7] highlighted the recent developments on fractal-based approaches to nanoparticle aggregation and nanofluids. Recently, Xia et al. [8] presented the optimization of a fractal-like architecture of porous medium associated with diffusivity, thermal conductivity, and permeability. They observed that the ratio of dimensionless permeability over dimensionless effective diffusivity diminished due to the decrement of tortuosity and porosity fractal dimensions. Hence, the lower tortuosity and porosity fractal dimensions were favorable to water/wind resistant fabric, as it is helpful to diminish the ratio of dimensionless permeability over dimensionless effective diffusivity. Further, they also found that the ratio of dimensionless total effective thermal conductivity over effective diffusivity enhances with tortuosity fractal dimension which reveals that the lower tortuosity fractal dimensional was helpful to clothing insulations, because it diminishes the ratio of dimensionless effective thermal conductivity over effective diffusivity. Some more pertinent studies on the said topic can be found from the references [9-13].

In a human body, symmetrical contraction and suspension of smooth muscles create a sinusoidal wave which is known as a peristaltic wave. It is a very well-known mechanism that helps in transporting various biological fluids, such as the transport of urine, in the human body. Chyme movement and vasomotion of small blood vessels are the common examples of peristaltic flow. Due to an extensive range of applications, peristaltic nanofluid flow grabbed the attention of different researchers. For instance, Bhatti et al. [14] discussed the peristaltic nanofluid flow in the presence of titanium nanoparticles with magnetic and endoscopic effects. He further obtained the series solutions using a homotopy perturbation method. Tripathi and Beg [15] presented a mathematical and theoretical study on nanofluids with peristalsis. They used the viscous fluid model and presented the exact solutions for the velocity, temperature, and nanoparticle concentration profiles. Akbar and Nadeem [16] considered PTT fluid model with nanoparticles propagating peristaltically in a diverging tube. Bhatti et al. [17] presented the two-phase flow simulation with heat and mass transfer in the presence of EDL ("Electric double layer") and MHD ("Magnetohydrodynamics") effects. A few more studies on the said topic can be found from the references [18-20].

Entropy generation can be defined as the phenomena of irreversibility processes of different thermal systems that are related to viscous dissipation, heat transfer, magnetic field and mass transfer etc. To enhance the performance of these irreversibilities, the second law of thermodynamics has been used [21-26] by various scientists. However, the first law of thermodynamics has also been used by various researchers and it is found that the second law of thermodynamic is more efficient and convenient as compared to the first law of thermodynamics. Rashidi et al. [27] investigated the entropy generation in steady magnetohydrodynamic flow of nanofluid over a rotating porous disk. Ranjit and Shit [28] recently investigated the entropy generation with a peristaltic flow in the presence of magnetic field. Akbar et al. [29] also discussed the entropy generation on peristaltic flow of water 
with copper nanoparticles. Sheikholeslami and Ganji [30] applied Lattice Boltzmann method (LBM) to determine the entropy generation on nanofluid flow with magnetic effects. Bhatti et al. [31,32] studied numerically the entropy generation with non-Newtonian fluid models through a shrinking and stretching sheet.

The purpose of the present study is to analyze the entropy generation on electro-kinetically modulated peristaltic propulsion on the magnetized nanofluid flow through a microchannel with joule heating. The effects of viscous dissipation, heat absorption, magnetic field, and electrokinetic body force are also taken into account. According to the best of our knowledge, no such analysis has been presented before.

\section{Mathematical Formulation}

The geometry model of the electro-osmotically peristaltic nanofluid flow through a microchannel is presented in Figure 1. The wavy wall surface of the microchannel is described as [33]:

$$
\widetilde{h}=\widetilde{a}-\widetilde{\phi} \cos ^{2} \pi \frac{(\widetilde{X}-\widetilde{c} \tilde{t})}{\lambda} .
$$

where $\widetilde{h}, \widetilde{a}, \widetilde{c}, \widetilde{t}, \widetilde{\phi}, \lambda$ are the transverse vibration of the wall, half width of the channel, velocity of the wave, time period, amplitude of the wave, and wave length, respectively. The temperature at the wall and the center line are presented as

$$
\begin{aligned}
& \widetilde{T}=\widetilde{T}_{0} \text { at } \widetilde{Y}=0, \\
& \widetilde{T}=\widetilde{T}_{1} \text { at } \widetilde{Y}=\widetilde{h} .
\end{aligned}
$$
as [17]:

The governing equations of motion, concentration, temperature, and continuity are expressed

$$
\begin{gathered}
\frac{\partial \widetilde{U}}{\partial \widetilde{X}}+\frac{\partial \widetilde{V}}{\partial \widetilde{Y}}=0 \\
\rho\left(\frac{\partial \widetilde{U}}{\partial \widetilde{t}}+\widetilde{U} \frac{\partial \widetilde{U}}{\partial \widetilde{X}}+\widetilde{V} \frac{\partial \widetilde{U}}{\partial \widetilde{Y}}\right)=-\frac{\partial \widetilde{P}}{\partial \widetilde{X}}+\mu\left(\frac{\partial^{2} \widetilde{U}}{\partial \widetilde{X}^{2}}+\frac{\partial^{2} \widetilde{U}}{\partial \widetilde{Y}}\right)+\rho \alpha g\left(\widetilde{T}-\widetilde{T}_{0}\right)-\sigma B_{0}^{2} \widetilde{U}+\rho_{e} E_{\widetilde{X}}, \\
\rho\left(\frac{\partial \widetilde{V}}{\partial \widetilde{t}}+\widetilde{U} \frac{\partial \widetilde{V}}{\partial \widetilde{X}}+\widetilde{V} \frac{\partial \widetilde{V}}{\partial \widetilde{Y}}\right)=-\frac{\partial \widetilde{P}}{\partial \widetilde{Y}}+\mu\left(\frac{\partial^{2} \widetilde{V}}{\partial \widetilde{X}{ }^{2}}+\frac{\partial^{2} \widetilde{V}}{\partial \widetilde{Y}}\right) \\
\rho c_{p}\left(\frac{\partial \widetilde{T}}{\partial \widetilde{t}}+\widetilde{U} \frac{\partial \widetilde{T}}{\partial \widetilde{X}}+\widetilde{V} \frac{\partial \widetilde{T}}{\partial \widetilde{Y}}\right)=\widetilde{k}\left(\frac{\partial^{2} \widetilde{T}}{\partial \widetilde{X}^{2}}+\frac{\partial^{2} \widetilde{T}}{\partial \widetilde{Y}}\right)+\mu\left(\frac{\partial \widetilde{U}}{\partial \widetilde{Y}}\right)^{2}+\Omega+\sigma E_{\widetilde{X}}^{2}+\sigma B_{0}^{2} \widetilde{U}^{2}
\end{gathered}
$$

With the help of Poisson Boltzmann equation, the electric potential with in a microchannel can be written as [17,33]:

$$
\nabla^{2} \widetilde{\Phi}=-\frac{\rho_{e}}{\varepsilon}
$$

where $\varepsilon$ is the permittivity, $\rho_{e}=e z\left[\widetilde{n}^{+}-\widetilde{n}^{-}\right]$is the electrical charge density, $e$ is the elementary charge, $\widetilde{n}^{+}$and $\widetilde{n}^{-}$are positive and negative charge ions consists of bulk concentration ("number density") $n_{0}$ and the valence of $z_{+}$and $z_{-}$respectively. Moreover, in order to analyze the potential distribution, it is compulsory to define the charge number density. For this purpose, Nernst-Planck equation is helpful to describe the number distributions of an individual species as [34].

$$
\left(\frac{\partial \widetilde{n}_{ \pm}}{\partial \widetilde{t}}+\widetilde{U} \frac{\partial \widetilde{n}_{ \pm}}{\partial \widetilde{X}}+\widetilde{V} \frac{\partial \widetilde{n}_{ \pm}}{\partial \widetilde{Y}}\right)=D\left(\frac{\partial^{2} \widetilde{n}_{ \pm}}{\partial \widetilde{X}^{2}}+\frac{\partial^{2} \widetilde{n}_{ \pm}}{\partial \widetilde{Y}^{2}}\right) \pm \frac{D z e}{\widetilde{T} k_{B}}\left[\frac{\partial}{\partial \widetilde{X}}\left(\widetilde{n}_{ \pm} \frac{\partial \widetilde{\Phi}}{\partial \widetilde{X}}\right)+\frac{\partial}{\partial \widetilde{Y}}\left(\widetilde{n}_{ \pm} \frac{\partial \widetilde{\Phi}}{\partial \widetilde{Y}}\right)\right]
$$


In the above equation, $D$ is the diffusivity of an ionic species, $k_{B}$ is the Boltzmann constant. The approximation of equal ionic diffusion coefficients for both species and the mobility of a species is described with the help of the Einstein formula.

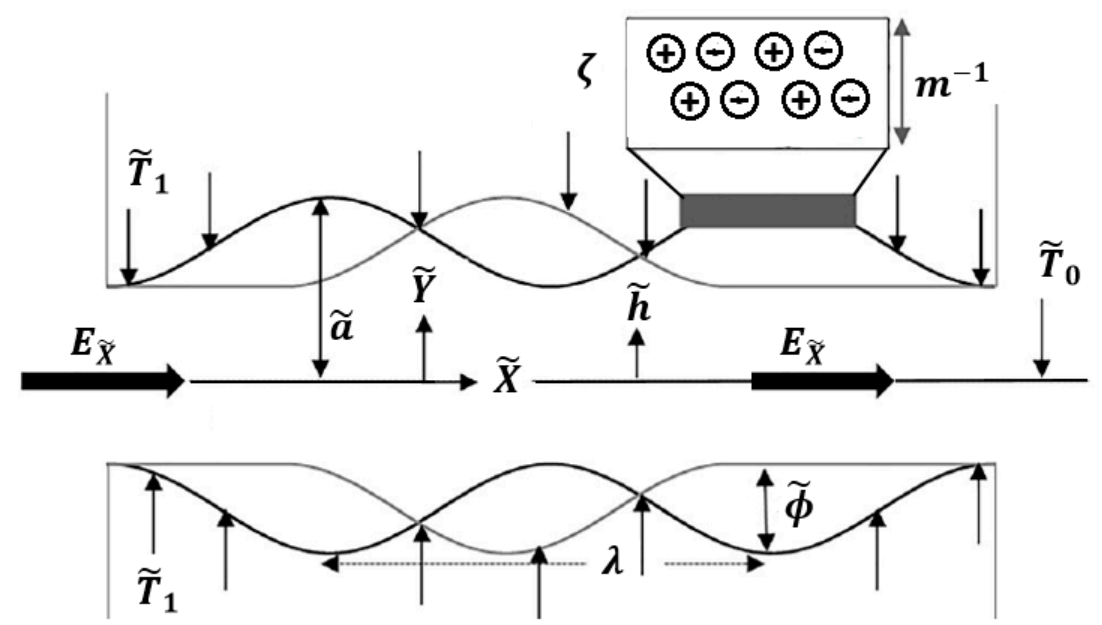

Figure 1. Flow structure of the electro-osmotically modulated peristaltic flow.

To move from the fixed frame to the wave frame, we define the following variables as $\widetilde{X}=$ $\bar{x}-\widetilde{c}, \bar{y}=\widetilde{Y}, \widetilde{U}-\widetilde{c}=\bar{u}, \widetilde{V}=\bar{v}$. Let us define the following non-dimensional quantities

$$
\begin{aligned}
& x=\frac{\bar{x}}{\lambda}, y=\frac{\bar{y}}{\widetilde{a}}, t=\frac{\widetilde{c} \widetilde{t}}{\lambda}, u=\frac{\bar{u}}{\tilde{c}}, v=\frac{\bar{w}}{\delta \widetilde{c}}, h=\frac{\widetilde{h}}{\widetilde{a}}, \phi=\frac{\widetilde{\Phi}}{\widetilde{a}} \delta=\frac{\widetilde{a}}{\lambda}, \Phi=\frac{\widetilde{\Phi}}{\zeta}, n=\frac{\widetilde{n}}{n_{0}} \\
& \theta=\frac{\widetilde{T}-\widetilde{T}_{0}}{\widetilde{T}_{1}-\widetilde{T}_{0}}, M^{2}=\frac{\sigma B_{0}^{2} \widetilde{a}^{2}}{\mu}, G r=\frac{g \alpha\left(\widetilde{T}_{0}-\widetilde{T}_{1}\right) \rho^{2} \widetilde{a}^{3}}{\mu^{2} \widetilde{c}}, \beta=\frac{\widetilde{a}^{2} \mu \Omega}{\left(\widetilde{T}_{1}-\widetilde{T}_{0}\right) \tilde{k}}, S c=\frac{\mu}{\rho D} \\
& S=\frac{\widetilde{a}^{2} E_{x}^{2} \sigma}{\left(\widetilde{T}_{1}-\widetilde{T}_{0}\right) \hat{k}^{k}}, U_{H S}=-\frac{E_{x} \zeta \varepsilon}{\mu \widetilde{c}}, B r=\frac{\widetilde{c}^{2} \mu}{\left(\widetilde{T}_{1}-\widetilde{T}_{0}\right) \vec{k}^{\prime}}, R e=\frac{\rho \delta \widetilde{c} a}{\mu}, \Omega_{T}=\frac{\widetilde{T}_{1}-\widetilde{T}_{0}}{\widetilde{T}_{0}}
\end{aligned}
$$

The nonlinear terms in Equation (9) are of $O\left(P e \delta^{2}\right)$, whereas $P e=S c R e$ is the ionic Peclet number and $S c$ denotes the Schmidt number. Therefore, the nonlinear terms dropped in the limit i.e., $R e, P e, \delta<<1$. The Poisson equation reduced into the following form

$$
\frac{\partial^{2} \Phi}{\partial y^{2}}+m^{2}\left[\frac{n_{+}-n_{-}}{2}\right]=0
$$

where $m=\tilde{a} e z \sqrt{2 n_{0} / k_{B} \varepsilon \widetilde{T}}=\widetilde{a} / \lambda_{d}$ is an electro-osmotic parameter and $\lambda_{d}=1 / m$ is debye length or characteristic thickness EDL ("electrical double layer"). After some simplification Equation (10), the ionic distribution can be written as

$$
\frac{\partial^{2} n_{ \pm}}{\partial y^{2}} \pm \frac{\partial}{\partial y}\left[n_{ \pm} \frac{\partial \Phi}{\partial y}\right]=0
$$

Along with following bulk conditions

$$
\left.\begin{array}{l}
n_{ \pm}(\Phi=0)=1 \\
\frac{\partial n_{ \pm}}{\partial y}=0, \text { and } \frac{\partial \Phi}{\partial y}=0 .
\end{array}\right\}
$$

Then the Boltzmann distributions for the ions are

$$
n_{ \pm}=e^{ \pm \Phi}
$$


Using Equations (11) and (14), we get

$$
\frac{\partial^{2} \Phi}{\partial y^{2}}=m^{2} \sinh \Phi
$$

To proceed further, Equation (15) must be modified by means of low-zeta potential approximation. This approximation is not beneficial due to a broad range of $\mathrm{pH}$ and the magnitude of the zeta potential is very small (less than $25 \mathrm{mV}$ ). Therefore, Equation (15) reduces to the following form

$$
\frac{\partial^{2} \Phi}{\partial y^{2}}=m^{2} \Phi
$$

Subject to

$$
\begin{aligned}
& \left.\frac{\partial \Phi}{\partial y}\right|_{y=0}=0, \\
& \left.\Phi\right|_{y=h}=1,
\end{aligned}
$$

whereas the potential function is defined as

$$
\Phi=\frac{\cosh m y}{\cosh m h}
$$

Using Equation (10) in Equations (1)-(7), and applying the approximation long wavelength and creeping flow regime,

$$
\begin{gathered}
\frac{\partial p}{\partial x}=\frac{\partial^{2} u}{\partial y^{2}}+G r \theta-M^{2}(u+1)+m^{2} U_{H S} \Phi \\
\frac{\partial p}{\partial y}=0 \\
\frac{\partial^{2} \theta}{\partial y^{2}}+S+\beta+B r\left[M^{2}(u+1)^{2}+\left(\frac{\partial u}{\partial y}\right)^{2}\right],
\end{gathered}
$$

subject to the following boundary conditions

$$
\frac{\partial u}{\partial y}=0, \theta=0 ; \text { at } y=0 \text { and } u=0, \theta=1 \text {; at } y=h
$$

\section{Entropy Generation Analysis}

The volumetric rate of a local entropy generation is described in the following form as:

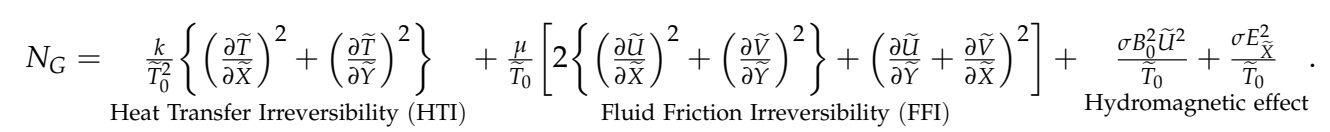

The above equation describes the dimensional form of entropy generation due to fluid friction irreversibility, heat transfer, and magnetic field. A characteristics entropy generation is defined as

$$
N_{G, 0}=\frac{k\left(\widetilde{T}_{1}-\widetilde{T}_{0}\right)^{2}}{\widetilde{T}_{0}^{2} \widetilde{a}^{2}} .
$$

Then the dimensionless form of entropy generation is written as

$$
N_{S}=\frac{N_{G}}{N_{G, 0}}=\left(\frac{\partial \theta}{\partial y}\right)^{2}+\frac{B r}{\Omega_{T}}\left(\frac{\partial u}{\partial y}\right)^{2}+\frac{M^{2} B r}{\Omega_{T}}(u+1)^{2}+\frac{S}{\Omega_{T}} .
$$


where $M, B r, \Omega_{T}$ are defined above. Furthermore, the integration of Equation (23) in the whole computational domains gives the dimensionless entropy generation, $N_{G}^{a v g}$, which can be written as

$$
N_{G}^{a v g}=\frac{1}{\chi} \int N_{G} d \chi
$$

Moreover, the Bejan number $B_{e}$ is a significant parameter that describes the importance of irreversibility process in a domain and can be described as

$$
B_{e}=\frac{\left(\frac{\partial \theta}{\partial y}\right)^{2}}{\left[\left(\frac{\partial \theta}{\partial y}\right)^{2}+\frac{B r}{\Omega_{T}}\left(\frac{\partial u}{\partial y}\right)^{2}+\frac{M^{2} B r}{\Omega_{T}}(u+1)^{2}+\frac{S}{\Omega_{T}}\right]} .
$$

The relative global supremacy of heat transfer irreversibility can be analyzed by average Bejan number $B_{e}^{a v g}$, which can be defined as

$$
B_{e}^{a v g}=\frac{N_{G, H T I}^{a v g}}{N_{G}^{a v g}} .
$$

It is noticeable here that $B_{e}^{a v g}<1 / 2$ reveals that the irreversibility process due to fluid friction dominates the flow whereas $B_{e}^{a v g}>1 / 2$ reveals that heat transfer irreversibility dominates and $B_{e}^{a v g}=1 / 2$ shows that the fluid friction and heat transfer entropy generation are equal.

\section{Solution of the Problem}

The exact solutions of Equations (19) and (21) are difficult to obtain due to a coupled nonlinearity. Therefore, we have applied the homotopy perturbation method $[17,25]$ to obtain the solutions. Therefore, the homotopy for Equations (19) and (21) are defined as:

$$
\begin{gathered}
H(\Theta, \xi)=(1-\xi)\left[L(\Theta)-L\left(\widetilde{\Theta}_{0}\right)\right]+\xi\left[L(\Theta)+G r \theta+m^{2} U_{H S} \Phi-M^{2}(u+1)-\frac{d p}{d x}\right], \\
H(\vartheta, \xi)=(1-\xi)\left[L(\vartheta)-L\left(\bar{\vartheta}_{0}\right)\right]+\xi\left[L(\vartheta)+\beta+S+B r\left(\frac{\partial u}{\partial y}\right)^{2}+M^{2} B r(u+1)^{2}\right],
\end{gathered}
$$

where $\xi$ is an embedding parameter. The Linear operator $L$ is considered as

$$
L=\frac{\partial^{2}}{\partial y^{2}}
$$

and the initial guesses for the above linear operators are defined as

$$
\widetilde{\Theta}_{0}=y^{2}-h^{2}, \bar{\vartheta}_{0}=\frac{y}{h}
$$

Defining the following expansions

$$
\begin{gathered}
\Theta(y)=\Theta_{0}(y)+\xi \Theta_{1}(y)+\xi^{2} \Theta_{2}(y)+\ldots, \\
\vartheta(y)=\vartheta_{0}(y)+\xi \vartheta_{1}(y)+\xi^{2} \vartheta_{2}(y)+\ldots .,
\end{gathered}
$$

Applying the series expansion in Equations (33)-(34) to the homotopy sets in Equations (29) and (30), then comparing the powers of $\xi$, the system of linear differential equations can be obtained. Using the property of homotopy perturbation scheme $[17,25]$ i.e., $\xi \rightarrow 1$, we have

$$
u(y)=\Theta(y)=\Theta_{0}(y)+\Theta_{1}(y)+\Theta_{2}(y)+\ldots,
$$




$$
\theta(y)=\vartheta(y)=\vartheta_{0}(y)+\vartheta_{1}(y)+\vartheta_{2}(y)+\ldots,
$$

The final form of solutions for the velocity profile and temperature can be written in simplified form as

$$
\begin{gathered}
u(y)=u_{0}+u_{1} y^{2}+u_{2} y^{3}+u_{3} y^{4}+u_{4} y^{5}+u_{5} y^{6}+u_{6} y^{8}+u_{7} \cosh m y \\
\theta(y)=\theta_{0}+\theta_{1} y+\theta_{2} y^{2}+\theta_{3} y^{4}+\theta_{4} y^{5}+\theta_{5} y^{6}+\theta_{6} y^{7}+\theta_{7} y^{8}+\theta_{8} \cosh m y+\theta_{9} y \sinh m y+\theta_{10} y^{2} \cosh m y,
\end{gathered}
$$

whereas the constant appearing i.e., $\left(u_{i}, \theta_{i} ; i=1,2,3 \ldots\right)$ in the above equations can be found using routine calculations. The instantaneous volume flow rate $Q$ can be calculated as

$$
Q=\int_{0}^{h} u \mathrm{~d} y .
$$

The mean flow rate over one period of wavelength can be written as

$$
\bar{Q}=Q+1-h-\frac{\phi}{2} .
$$

The pressure rise is evaluated numerically with the help of the following expression

$$
\Delta p=\int_{0}^{x} \frac{d p}{d z} \mathrm{~d} z
$$

\section{Results and Discussion}

The main idea of this section is to provide the physical behavior of all the governing parameters that are included in the present flow problem. To determine graphically, a symbolic computational software "Mathematica" has been utilized. For this purpose, Figure 2 to Figure 12 are sketched against velocity profile, temperature profile, Bejan number. and entropy profile for the following parameters i.e., Hartmann number $M$, thermal Grashof number $G r$, electro-osmotic parameter $m$, Helmholtz-Smoluchowski velocity (or "maximum electro-osmotic velocity") $U_{H S}$, Joule heating parameter $S$, heat source/sink $\beta$, amplitude ratio $\phi$, and Brinkmann number $B r$. Pumping features have also been evaluated numerically with the help of Equation (33) and are discussed. Figure 2 shows the numerical comparison with previously published results [35] by ignoring the magnetic field $(M \rightarrow 0)$ and the viscous dissipation effects in the governing equations. From this figure, we can see that the present results are in excellent agreement with previously published data. Therefore, it is concluded that the methodology used to obtain the results are correct and the present results converge. In the all the upcoming figures, the red, blue, and green colors reveal the variation of the mentioned variable in ascending order. However, the solid and dashed lines indicate the lower to the higher value for the given parameter.

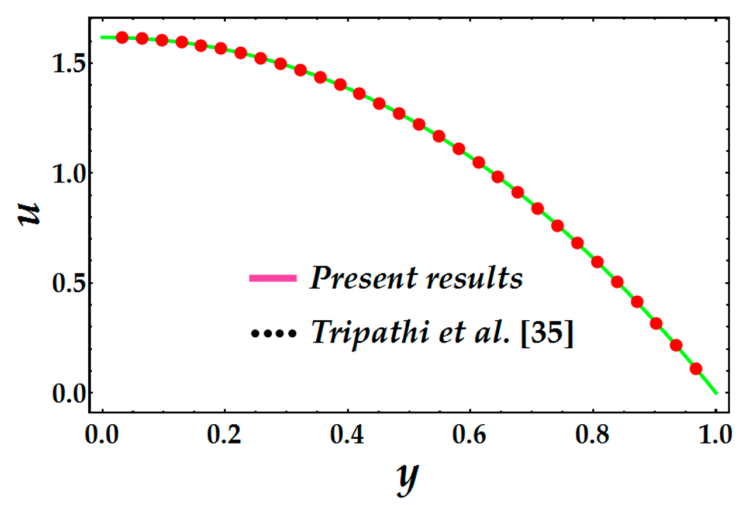

Figure 2. Comparison of velocity distribution with previously published results [35]. 
Figure 3 represents the variation of the velocity profile against different values of thermal Grashof number $G r$ and Hartmann number $M$. In this figure we can see that the thermal Grashof number significantly enhances the velocity profile in the region $y \in[0.5,1]$, however, the results are completely converse when $y<0.5$. On the other hand, the effects of Hartmann number $M$ is completely opposite as compared to thermal Grashof number. The velocity of the fluid tends to diminish when $y>0.5$, because when the magnetic field is applied to any conducting fluid, a converse force occurs which tends to oppose the velocity and provide a marked resistance in the flow field. Figure 3 is plotted for electro-osmotic parameter $m$, and Helmholtz-Smoluchowski velocity (or "maximum electro-osmotic velocity") $U_{H S}$. In Figure 4 , we can see that the electro-osmotic parameter accelerates the fluid velocity in the region $y \in[0.5,1]$, whereas its behavior becomes the opposite for $y<0.5$. Electro-osmotic parameter is the ratio of channel length and a Debye length $m\left(=\widetilde{a} / \lambda_{d}\right)$ which reveals that the increment in the height of the channel produces a significant enhancement in velocity profile and consequently and increment in electrical Debye length. Further, Helmholtz-Smoluchowski velocity (or "maximum electro-osmotic velocity") $U_{H S}\left(=-E_{x} \zeta \varepsilon / \mu \widetilde{\mathcal{C}}\right)$ also boosts the velocity profile in the similar region as compared to the electro-osmotic parameter.

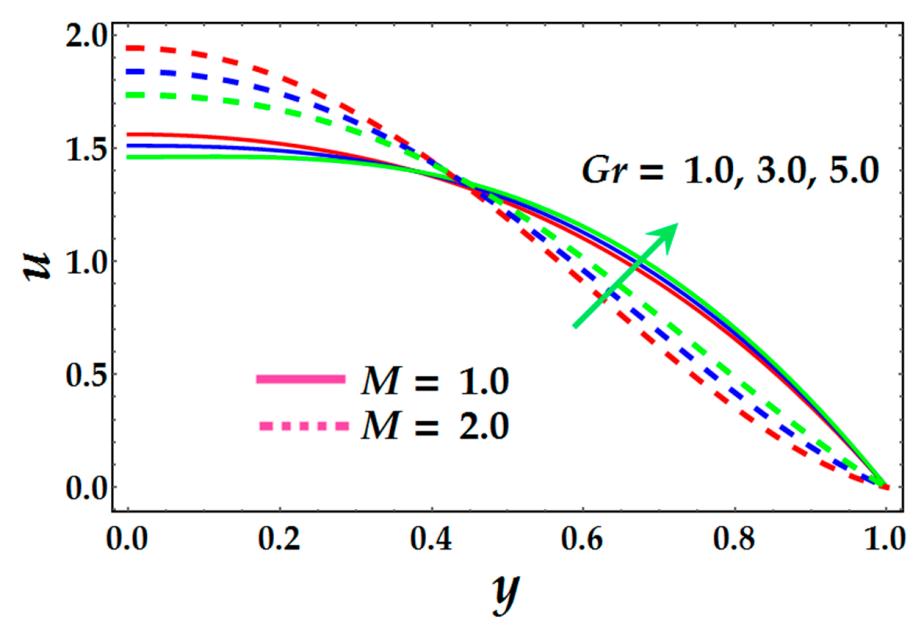

Figure 3. Velocity distribution against multiple values of $M$ and $G r$.

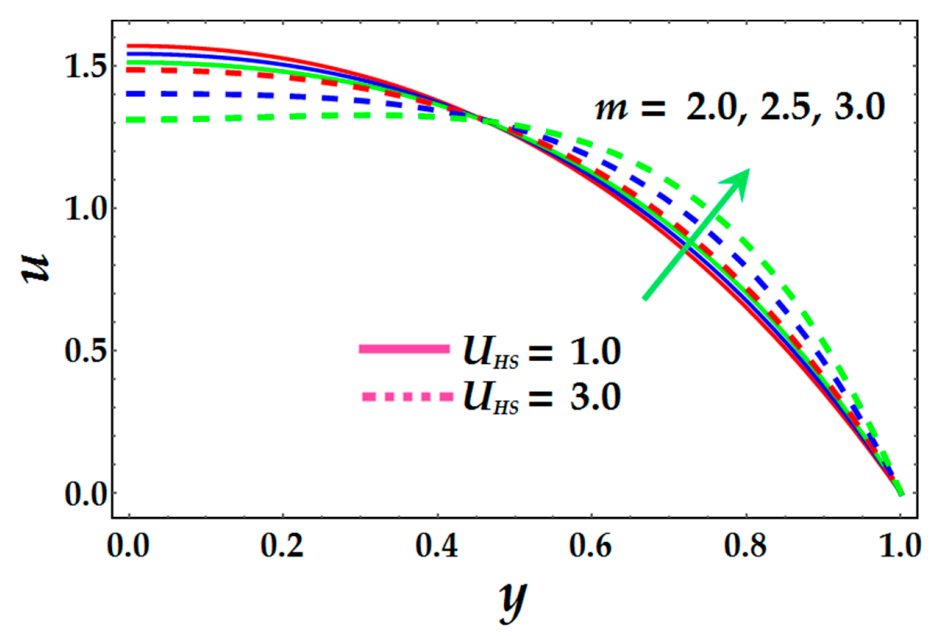

Figure 4. Velocity distribution against multiple values of $U_{H S}$ and $m$.

Figures 5 and 6 represent the temperature profile against different values of Hartmann number $M$, Joule heating parameter $S$, heat source/sink $\beta$, and Brinkmann number $B r$. It can be noticed from Figure 4 that Joule heating parameter $S$, significantly raises the temperature profile. The Joule heating 
parameter $S$ is a normalized generation term that shows the ratio of Joule heating to the surface heat flux. The result is cooling in the electrokinetic flow through a microchannel with a negative $S$ and heating with a positive $S$. Further, Joule heating is related to the electric field induced by the internal heating process in a fluid in the presence of an applied potential gradient. Higher values of an electric field cause a higher heat generation which results in heating of the fluid. In Figure 5 we can further notice that higher values of heat source/sink $\beta$, produce a positive effect on the temperature profile and significantly boosts the temperature profile. Figure 6 shows that the effects of magnetic field and Brinkmann number accelerate the temperature profile. In fact, an increment in Brinkmann number tends to diminish the conduction of heat that occurs due to viscous dissipation and, as a result, the temperature rises significantly.

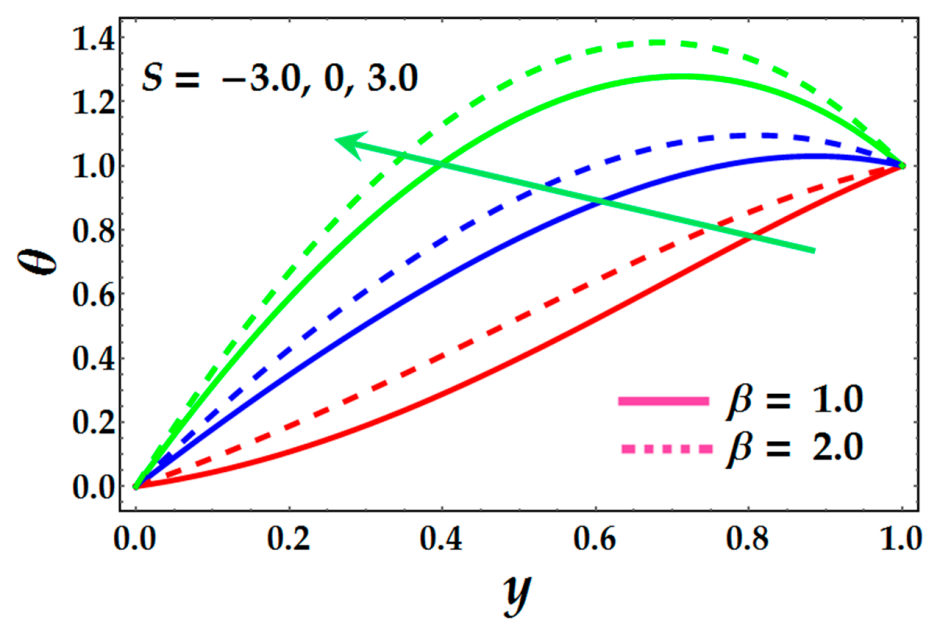

Figure 5. Temperature distribution against multiple values of $\beta$ and $S$.

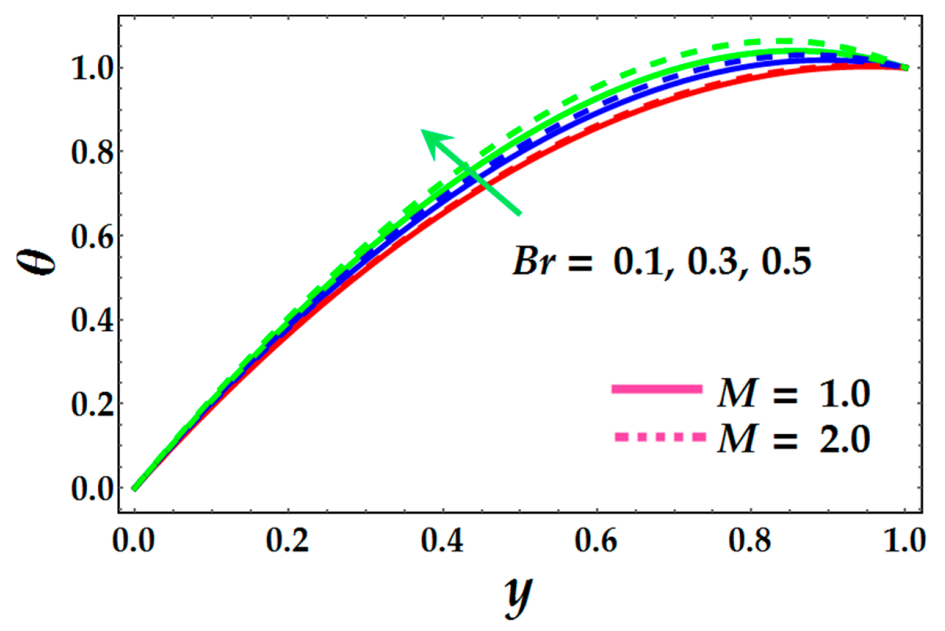

Figure 6. Temperature distribution against multiple values of $\mathrm{M}$ and $\mathrm{Br}$.

Figures 7 and 8 are sketched to analyze the behavior of peristaltic pumping in different pumping regions. It is a very important and substantial part of the peristaltic flow that is beneficial in the propagation of multiple fluids. It can be observed from Figure 7 that the Hartmann number $M$, and Helmholtz-Smoluchowski velocity (or "maximum electro-osmotic velocity") $U_{H S}$ behaves in a similar way on pressure rise. For instance, with the increment of both the parameters $U_{H S}, M$, the pumping rate increases in the retrograde pumping region $[\Delta p>0, Q<0]$, whereas the behavior of pumping rate changes in peristaltic pumping $[\Delta p>0, Q>0]$. and becomes opposite in co-pumping region $[\Delta p<0, Q>0]$. It can be viewed from Figure 8 that an enhancement in the electro-osmotic parameter 
$m$, creates a noticeable increment in the peristaltic pumping region and retrograde pumping, whereas its attitude is completely opposite in the co-pumping region. Further we can see that amplitude ratio $\phi$ acts similarly to an electro-osmotic parameter in all the regions but it has a greater impact on pressure rise.

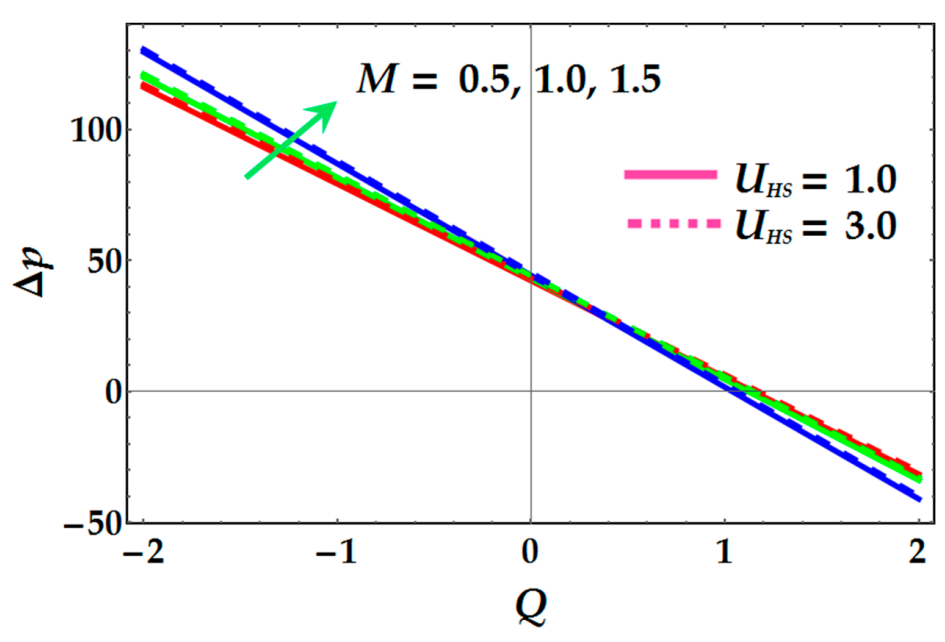

Figure 7. Pressure distribution against multiple values of $M$ and $U_{H S}$.

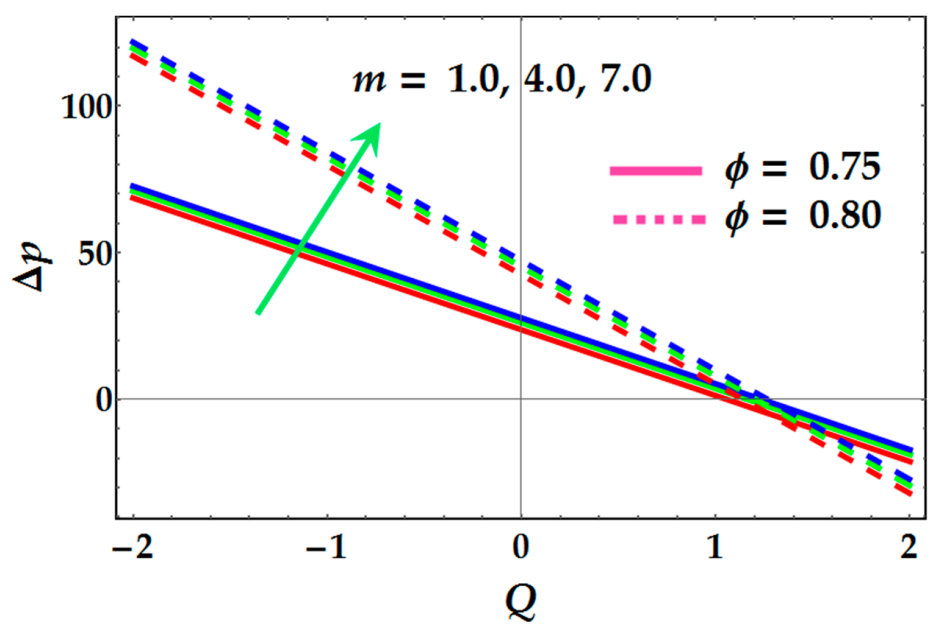

Figure 8. Pressure distribution against multiple values of $m$ and $\phi$.

Figures 9 and 10 are sketched to determine the behavior of entropy profile against different values of Hartmann number $M$, temperature difference $\Omega_{T}$, Joule heating parameter $S$, and Brinkmann number $B r$. Figure 9 shows that an increment in Hartmann number $M$ causes a significant increment in the entropy profile and remains positive throughout the region, whereas the parameter $\Omega_{T}$ shows opposite attitude on the entropy profile. It can be seen from Figure 10 that when the Joule heating parameter $S$, and Brinkmann number $\mathrm{Br}$ increase, then the entropy profile greatly increases and it remains positive throughout the region. Figures 11 and 12 show the Bejan number variation along multiple values of Hartmann number $M$, temperature difference $\Omega_{T}$, Joule heating parameter $S$, and Brinkmann number $B r$. From both the figures, one can easily observe that an increment in Hartmann number $M$, and Joule heating parameter $S$, and Brinkmann number $B r$ tends to diminish the Bejan number significantly, however, converse behavior has been observed against the temperature difference $\Omega_{T}$. 


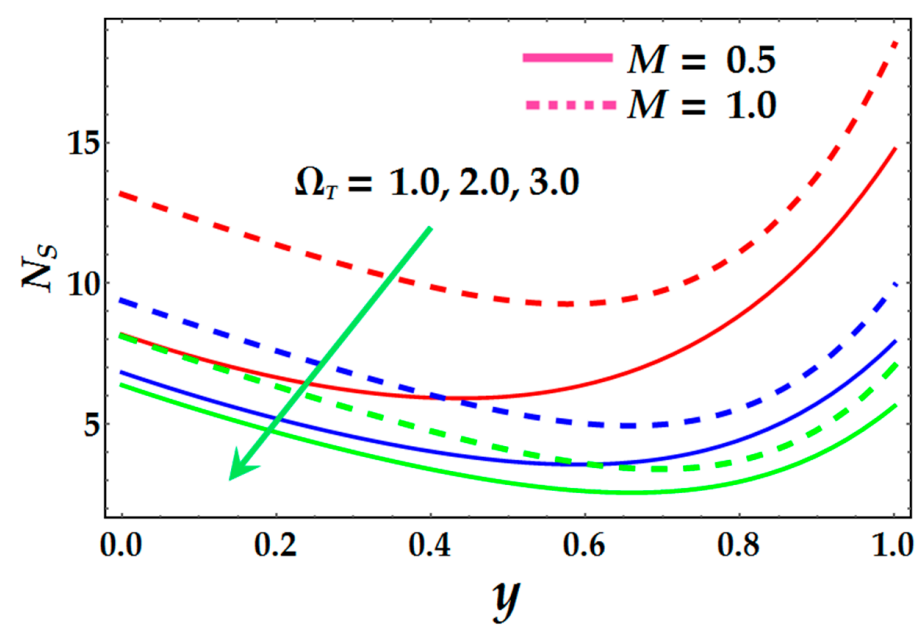

Figure 9. Entropy profile against multiple values of $M$ and $\Omega_{T}$.

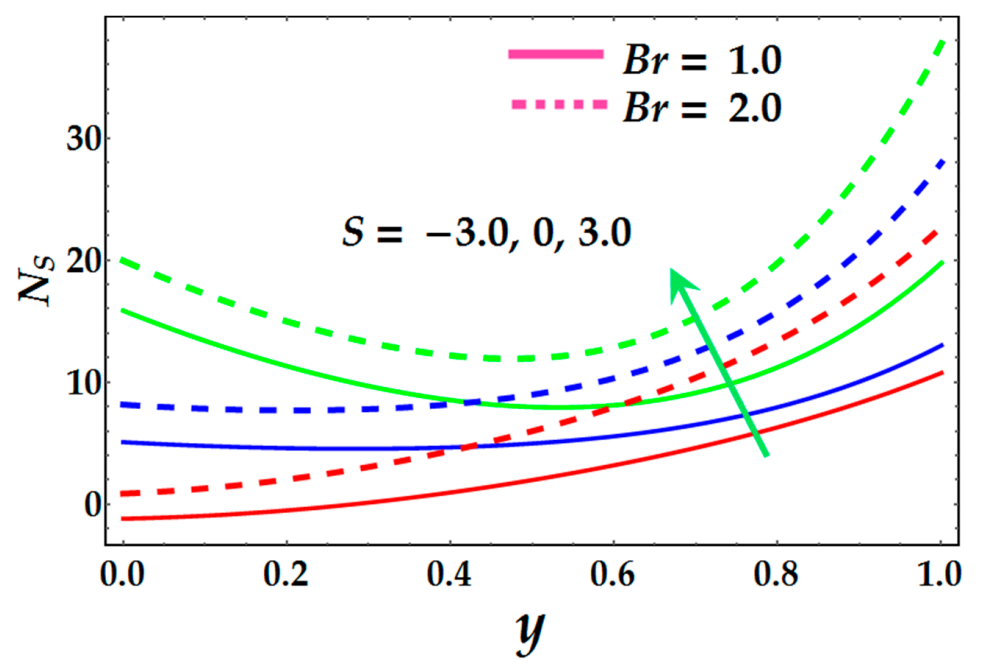

Figure 10. Entropy profile against multiple values of $S$ and $B r$.

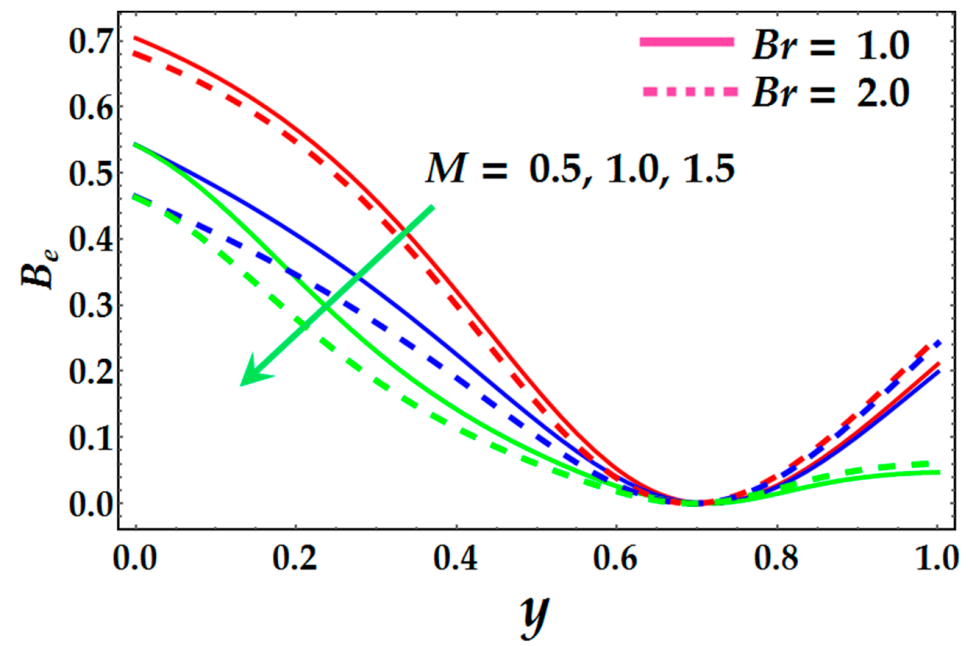

Figure 11. Variation of Bejan number against multiple values of $M$ and $B r$. 


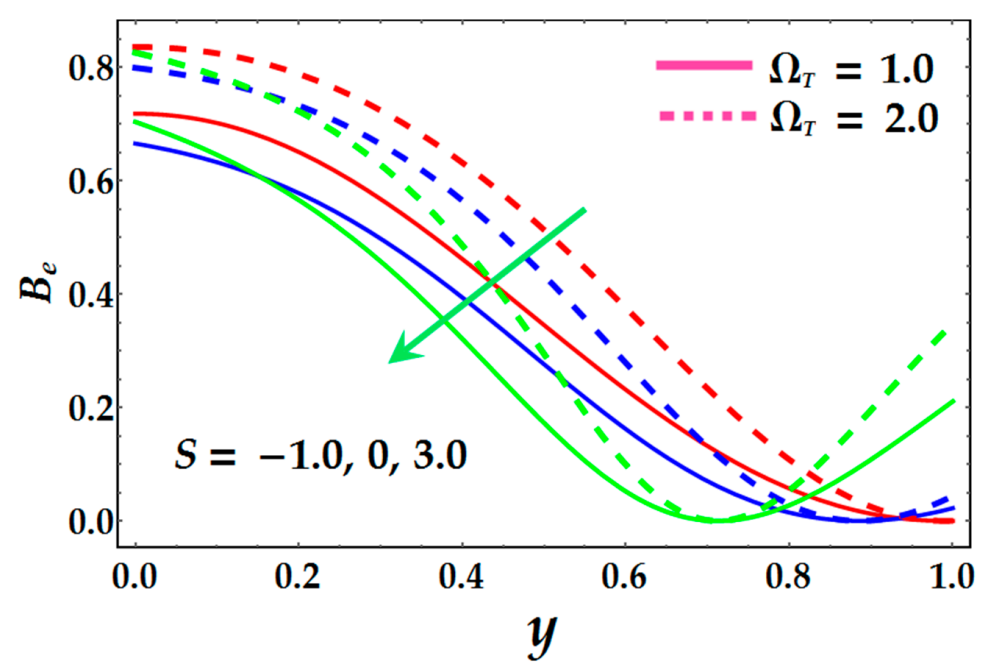

Figure 12. Variation of Bejan number against multiple values of $S$ and $\Omega_{T}$.

\section{Conclusions}

The present study deals with the theoretical study of entropy generation on electro-kinetically modulated peristaltic propulsion on magnetized nanofluid flow through a microchannel with joule heating. The effects of heat absorption, viscous dissipation, electrokinetic body force, and magnetic field are also considered in the present flow. Series solutions have been obtained for the velocity profile and temperature profile, whereas numerical integration has been used to evaluate the expression for pressure. The major findings for the governing flow problem are as follows:

i. The velocity of the fluid behaves in a similar manner due to the increment in thermal Grashof number and magnetic field.

ii. The velocity profile increases when $y>0.5$ for higher values of electro-osmotic parameter and Helmholtz-Smoluchowski velocity (or "maximum electro-osmotic velocity").

iii. The temperature profile reveals a significant increment due to the higher presence of Brinkmann number and magnetic field.

iv. An increment in the Joule heating parameter and heat source/sink significantly accelerates the temperature profile.

v. Pressure rise exhibits similar behavior to higher values of an electro-osmotic parameter, magnetic field, and Helmholtz-Smoluchowski velocity (or "maximum electro-osmotic velocity").

vi. The entropy profile also shows a positive response due to the greater impact of Brinkmann number, magnetic field, and the Joule heating parameter.

Author Contributions: The authors wrote all parts of this paper together.

Conflicts of Interest: The authors declare no conflict of interest.

\section{Nomenclature}

$\begin{array}{ll}\widetilde{h} & \text { Transverse vibration of the wall } \\ \widetilde{a} & \text { Half width of the channel } \\ B_{e} & \text { Local Bejan number } \\ B_{e}^{a v g} & \text { Average Bejan number } \\ \widetilde{P} & \text { Pressure } \\ \widetilde{c} & \text { Velocity of the wave } \\ \operatorname{Re} & \text { Reynolds number }\end{array}$




$\begin{array}{ll}N_{S} & \text { Dimensionless entropy number } \\ \widetilde{t} & \text { Time } \\ \widetilde{U}, \widetilde{V} & \text { Velocity field } \\ E_{\widetilde{X}} & \text { Electrokinetic body force } \\ \widetilde{k} & \text { Thermal conductivity } \\ \widetilde{X}, \widetilde{Y} & \text { Cartesian coordinate axis } \\ D & \text { Diffusivity of an ionic species } \\ k_{B} & \text { Boltzmann constant } \\ m & \text { Electroosmosis parameter } \\ S & \text { Joule heating parameter } \\ U_{H S} & \text { Helmholtz-Smoluchowski velocity } \\ B r & \text { Brinkman number } \\ G r & \text { Grashof number } \\ \bar{Q} & \text { Average volume flow rate } \\ M & \text { Hartman number } \\ B_{0} & \text { Magnetic field } \\ Q & \text { Volume flow rate } \\ \widetilde{T} & \text { Temperature } \\ g & \text { Acceleration due to gravity } \\ n_{0} & \text { Bulk concentration (number density) } \\ e & \text { Elementary charge }\end{array}$

\section{Greek Symbol}

$\begin{array}{ll}\rho & \text { Density of the fluid } \\ \alpha & \text { Coefficient of linear thermal expansion of fluid } \\ \Omega & \text { Heat absorption coefficient } \\ \mu & \text { Viscosity of the fluid } \\ \varepsilon & \text { Permittivity } \\ \lambda_{d} & \text { Debye length } \\ \lambda & \text { Wave length } \\ \rho_{e} & \text { Electrical charge density } \\ \widetilde{\phi} & \text { Amplitude of the wave } \\ \theta & \text { Temperature profile } \\ \xi & \text { Embedding parameter } \\ \sigma & \text { Electrical conductivity } \\ \Omega_{T} & \text { Dimensionless temperature difference } \\ \beta & \text { Dimensionless heat source/sink } \\ \delta & \text { Wave number }\end{array}$

\section{References}

1. Choi, S.U.S. Enhancing thermal conductivity of fluids with nanoparticles. ASME Fed 1995, 231, 99-106.

2. Khanafer, K.; Vafai, K.; Lightstone, M. Buoyancy-driven heat transfer enhancement in a two-dimensional enclosure utilizing nanofluids. Int. J. Heat Mass Transf. 2003, 46, 3639-3653. [CrossRef]

3. Oztop, H.F.; Abu-Nada, E. Numerical study of natural convection in partially heated rectangular enclosures filled with nanofluids. Int. J. Heat Fluid Flow 2008, 29, 1326-1336. [CrossRef]

4. Sheremet, M.A.; Pop, I. Natural convection in a wavy porous cavity with sinusoidal temperature distributions on both side walls filled with a nanofluid: Buongiorno's mathematical model. J. Heat Transf. 2015, 137, 072601. [CrossRef]

5. Xiao, B.; Yang, Y.; Chen, L. Developing a novel form of thermal conductivity of nanofluids with Brownian motion effect by means of fractal geometry. Powder Technol. 2013, 239, 409-414. [CrossRef] 
6. Sheremet, M.A.; Pop, I.; Bachok, N. Effect of thermal dispersion on transient natural convection in a wavy-walled porous cavity filled with a nanofluid: Tiwari and Das' nanofluid model. Int. J. Heat Mass Transf. 2016, 92, 1053-1060. [CrossRef]

7. Cai, J.; Hu, X.; Xiao, B.; Zhou, Y.; Wei, W. Recent developments on fractal-based approaches to nanofluids and nanoparticle aggregation. Int. J. Heat Mass Transf. 2017, 105, 623-637. [CrossRef]

8. Xiao, B.; Wang, W.; Fan, J.; Chen, H.; Hu, X.; Zhao, D.; Zhang, X.; Ren, W. Optimization of the fractal-like architecture of porous fibrous materials related to permeability, diffusivity and thermal conductivity. Fractals 2017, 25, 1750030. [CrossRef]

9. Sheikholeslami, M. Magnetic field influence on $\mathrm{CuO}-\mathrm{H}_{2} \mathrm{O}$ nanofluid convective flow in a permeable cavity considering various shapes for nanoparticles. Int. J. Hydrog. Energy 2017, 42, 19611-19621. [CrossRef]

10. Sheikholeslami, M.; Bhatti, M.M. Active method for nanofluid heat transfer enhancement by means of EHD. Int. J. Heat Mass Transf. 2017, 109, 115-122. [CrossRef]

11. Sheikholeslami, M.; Bhatti, M.M. Forced convection of nanofluid in presence of constant magnetic field considering shape effects of nanoparticles. Int. J. Heat Mass Transf. 2017, 111, 1039-1049. [CrossRef]

12. Sheikholeslami, M. Lattice Boltzmann Method simulation of MHD non-Darcy nanofluid free convection. Phys. B Cendens. Matter 2017, 516, 55-71. [CrossRef]

13. Ellahi, R.; Mubashir Bhatti, M.; Riaz, A.; Sheikholeslami, M. Effects of magnetohydrodynamics on peristaltic flow of Jeffrey fluid in a rectangular duct through a porous medium. J. Porous Media 2014, 17, 143-157. [CrossRef]

14. Bhatti, M.M.; Zeeshan, A.; Ellahi, R. Endoscope analysis on peristaltic blood flow of Sisko fluid with Titanium magneto-nanoparticles. Comput. Biol. Med. 2016, 78, 29-41. [CrossRef] [PubMed]

15. Tripathi, D.; Bég, O.A. A study on peristaltic flow of nanofluids: Application in drug delivery systems. Int. J. Heat Mass Transf. 2014, 70, 61-70. [CrossRef]

16. Akbar, N.S.; Nadeem, S. Peristaltic flow of a Phan-Thien-Tanner nanofluid in a diverging tube. Heat Transf. Asian Res. 2012, 41, 10-22. [CrossRef]

17. Bhatti, M.M.; Zeeshan, A.; Ellahi, R.; Ijaz, N. Heat and mass transfer of two-phase flow with Electric double layer effects induced due to peristaltic propulsion in the presence of transverse magnetic field. J. Mol. Liq. 2017, 230, 237-246. [CrossRef]

18. Sheikholeslami, M.; Rokni, H.B. Simulation of nanofluid heat transfer in presence of magnetic field: A review. Int. J. Heat Mass Transf. 2017, 115, 1203-1233. [CrossRef]

19. Akbar, N.S.; Raza, M.; Ellahi, R. Copper oxide nanoparticles analysis with water as base fluid for peristaltic flow in permeable tube with heat transfer. Comput. Methods Progr. Biomed. 2016, 130, 22-30. [CrossRef] [PubMed]

20. Abbasi, F.M.; Hayat, T.; Ahmad, B. Peristaltic transport of copper-water nanofluid saturating porous medium. Phys. E 2015, 67, 47-53. [CrossRef]

21. Bejan, A. Entropy Generation Minimization: The Method of Thermodynamic Optimization of Finite-Size Systems and Finite-Time Processes; CRC Press: Boca Raton, FL, USA, 1996.

22. Bejan, A. Second-law analysis in heat transfer and thermal design. Adv. Heat Trans. 1982, 15, 1-58.

23. Bejan, A. Second law analysis in heat transfer. Energy 1980, 5, 720-732. [CrossRef]

24. Rashidi, M.M.; Bhatti, M.M.; Abbas, M.A.; Ali, M.E.S. Entropy generation on MHD blood flow of nanofluid due to peristaltic waves. Entropy 2016, 18, 117. [CrossRef]

25. Abbas, M.A.; Bai, Y.; Rashidi, M.M.; Bhatti, M.M. Analysis of entropy generation in the flow of peristaltic nanofluids in channels with compliant walls. Entropy 2016, 18, 90. [CrossRef]

26. Sheremet, M.A.; Oztop, H.F.; Pop, I.; Abu-Hamdeh, N. Analysis of entropy generation in natural convection of nanofluid inside a square cavity having hot solid block: Tiwari and Das' model. Entropy 2015, 18, 9. [CrossRef]

27. Rashidi, M.M.; Abelman, S.; Mehr, N.F. Entropy generation in steady MHD flow due to a rotating porous disk in a nanofluid. Int. J. Heat Mass Trans. 2013, 62, 515-525. [CrossRef]

28. Ranjit, N.K.; Shit, G.C. Entropy generation on electro-osmotic flow pumping by a uniform peristaltic wave under magnetic environment. Energy 2017, 128, 649-660. [CrossRef]

29. Akbar, N.S.; Raza, M.; Ellahi, R. Peristaltic flow with thermal conductivity of $\mathrm{H}_{2} \mathrm{O}^{+} \mathrm{Cu}$ nanofluid and entropy generation. Results Phys. 2015, 5, 115-124. [CrossRef] 
30. Sheikholeslami, M.; Ganji, D.D. Entropy generation of nanofluid in presence of magnetic field using Lattice Boltzmann Method. Physica A 2015, 417, 273-286. [CrossRef]

31. Bhatti, M.M.; Abbas, T.; Rashidi, M.M.; Ali, M.E.S. Numerical simulation of entropy generation with thermal radiation on MHD Carreau nanofluid towards a shrinking sheet. Entropy 2016, 18, 200. [CrossRef]

32. Bhatti, M.M.; Abbas, T.; Rashidi, M.M.; Ali, M.E.S.; Yang, Z. Entropy generation on MHD Eyring-Powell nanofluid through a permeable stretching surface. Entropy 2016, 18, 224. [CrossRef]

33. Tripathi, D.; Bhushan, S.; Bég, O.A. Transverse magnetic field driven modification in unsteady peristaltic transport with electrical double layer effects. Colloids Surf. A 2016, 506, 32-39. [CrossRef]

34. Sokalski, T.; Lingenfelter, P.; Lewenstam, A. Numerical solution of the coupled Nernst-Planck and Poisson equations for liquid junction and ion selective membrane potentials. J. Phys. Chem. B 2003, 107, 2443-2452. [CrossRef]

35. Tripathi, D.; Sharma, A.; Bég, O.A.; Tiwari, A. Electrothermal Transport in Biological Systems: An Analytical Approach for Electrokinetically Modulated Peristaltic Flow. J. Therm. Sci. Eng. Appl. 2017, 9, 041010. [CrossRef]

(C) 2017 by the authors. Licensee MDPI, Basel, Switzerland. This article is an open access article distributed under the terms and conditions of the Creative Commons Attribution (CC BY) license (http://creativecommons.org/licenses/by/4.0/). 